\title{
High-Fidelity Multidisciplinary Design Optimization Methodology with Application to Rotor Blades
}

\author{
Li Wang \\ Senior Research Engineer \\ National Institute of Aerospace \\ Hampton, VA, U.S.A. \\ Eric J. Nielsen \\ Research Scientist \\ NASA Langley Research Center \\ Hampton, VA, U.S.A.
}

\author{
Boris Diskin \\ Research Fellow \\ National Institute of Aerospace \\ Hampton, VA, U.S.A. \\ Valentin Sonneville \\ Assistant Research Scientist \\ University of Maryland \\ College Park, MD, U.S.A.
}

\author{
Robert T. Biedron \\ Research Scientist \\ NASA Langley Research Center \\ Hampton, VA, U.S.A. \\ Olivier A. Bauchau \\ Professor \\ University of Maryland \\ College Park, MD, U.S.A.
}

\begin{abstract}
A multidisciplinary design optimization procedure has been developed and applied to rotorcraft simulations involving tightly-coupled, high-fidelity computational fluid dynamics and comprehensive analysis. A discretely-consistent, adjoint-based sensitivity analysis available in the fluid dynamics solver provides sensitivities arising from unsteady turbulent flows on unstructured, dynamic, overset meshes, while a complex-variable approach is used to compute structural sensitivities with respect to aerodynamic loads. The multidisciplinary sensitivity analysis is conducted through integrating the sensitivity components from each discipline of the coupled system. Accuracy of the coupled system for high-fidelity rotorcraft analysis is verified; simulation results exhibit good agreement with established solutions. A constrained gradient-based design optimization for a HART-II rotorcraft configuration is demonstrated. The computational cost for individual components of the multidisciplinary sensitivity analysis is assessed and improved.
\end{abstract}

\section{INTRODUCTION}

Accurate analysis of rotorcraft aeromechanics requires many disciplines such as aerodynamics, aeroacoustics, structural dynamics and deformations, flight mechanics, and others to capture complex interactions of unsteady flows with highly flexible rotor blades. Multidisciplinary rotorcraft comprehensive analysis (CA) tools (Refs. 1-3) are often used to simulate rotorcraft aeromechanics. These tools are computationally efficient, but rely on low-fidelity aerodynamic models, such as lifting-line and vortex-wake models that depend on either linear methods or experimental data corrections. To account for complicated three-dimensional effects and compressibility of the flow field, state-of-the-art high-fidelity rotorcraft simulations couple a CA model with a physics-based computational fluid dynamics (CFD) model (Refs. 4-7).

Gradient-based optimization of rotorcraft simulations has been a focus of the aeromechanics community in the past 20 years (Refs. 8,9). Most of the studies involving CA tools have been conducted using finite-difference approximations for sensitivities. Finite-difference approaches are effective for computing sensitivity of many objective functions with respect to a few design parameters. For a high-fidelity rotorcraft analysis, a single large-scale simulation of a coupled CFD/CA system may require hundreds of thousands of CPU hours. For optimization involving such simulations and many design parameters, finite-difference sensitivity analysis is not feasible. On the other hand, adjoint formulations developed in some state-of-the-art CFD solvers enable efficient computations of sensitivity of an objective function to many design parameters (Refs. 10,11). The computational cost of an adjoint-based sensitivity analysis approximately equals the cost of a single simulation and does not increase with the number of design parameters. This property makes adjoint methods especially suitable for shape optimization where a single or a few objective/constraint functions are used, but the number of design parameters is large.

This paper presents an adjoint-based multidisciplinary design optimization approach for high-fidelity CFD/CA simulations based on a tight-coupling methodology. An unstructured-grid, highly-scalable CFD solver, FUN3D (Refs. 5, 12), and a nonlinear flexible multibody dynamics CA solver, DYMORE (Ref. 3), are coupled to predict the airloads and structural responses of helicopter rotor blades. A discretely-consistent, adjoint-based sensitivity analysis available in FUN3D provides sensitivities arising from unsteady turbulent flows on unstructured, dynamic, overset meshes, while a complex-variable approach (Ref. 13) is used to compute sensitivities of DYMORE solutions with respect to aerodynamic loads. The multidisciplinary sensitivity analysis integrates all sensitivity components of the coupled system.

The focus of this work is on the multidisciplinary sensitivity analysis and performance improvements of the CFD/CA system. Previously, Refs. 7,14 and 15 reported on coupling of earlier DYMORE models with FUN3D for analysis and design optimization. A new comprehensive analysis model, 
denoted as DYMORE5, is used in the current work. This new model employs a local-frame motion formalism (Refs. 16-18) for finite-element simulation of rotor dynamics. The motion formalism reduces nonlinearity of the motion equations and results in nearly constant iteration matrices, thereby avoiding costly factorization at each time step. Furthermore, a domain decomposition technique has been developed in DYMORE5, which enables the use of OpenMP and Message Passing Interface libraries for parallel computing. These DYMORE5 capabilities lead to significant reductions in computing time without compromising accuracy.

The rest of the paper is organized as follows. The next section describes the CFD and CA solvers used in this work and mathematical formulations of the multidisciplinary sensitivity analysis. Then the coupled CFD/CA system is verified through comparisons of the FUN3D/DYMORE5 simulations with established solutions for a HART-II rotorcraft test model. A constrained gradient-based design optimization is then demonstrated, and the computational efficiency is analyzed.

\section{TECHNICAL APPROACH}

In this section, the CFD and CA solvers used in this study are outlined. Mathematical formulations for the CFD/CA analysis and multidisciplinary sensitivity analysis are given, and the design optimization procedure is described.

\section{Flow and Comprehensive Analysis Solvers}

Solutions of the Reynolds-averaged Navier-Stokes (RANS) equations are computed with the FUN3D flow solver (Refs. 5, 12) developed and supported by the NASA Langley Research Center. FUN3D is a finite-volume, node-centered, unstructured-grid RANS solver, which is widely used for high-fidelity analysis and adjoint-based optimization of complex turbulent flows (Refs. 10,19). To advance the flow equations in time, a library of time integration schemes is available including standard first-order and second-order backward difference schemes known as BDF1 and BDF2 schemes, respectively, as well as a blended second- and third-order backward difference scheme, referred to as BDF2opt (Ref. 20). For overset meshes, the DiRTlib (Ref. 21) and SUGGAR++ (Ref. 22) codes are used to facilitate communications between components of the mesh.

The CA code used in the current study is a nonlinear flexible multibody dynamics code, DYMORE (Ref. 3), which provides static, dynamic, stability, and trim analyses of rotorcraft configurations. DYMORE contains libraries of primitive elements such as rigid bodies, mechanical joints, elastic springs, dampers, beams, shells, and plates. The internal aerodynamics model in DYMORE is a low-fidelity approximation based on lifting-line theory. For this study, the new DYMORE5 model (Refs. 16-18) has been employed. DYMORE5 is capable of operating in both real and complex modes. The complex-mode operation is achieved by replacing all realvalued variables in the code with complex-valued variables and overwriting the real-valued functions and operators with corresponding complex-valued functions and operators. Sensitivities of DYMORE5 solutions can be evaluated through complex-variable analysis (Refs. 13,14). Unlike a real-valued finite-difference approach, the complex-variable perturbation calculations do not suffer from inaccuracies due to subtractive cancellation errors (Refs. 13, 23, 24), thereby providing increased accuracy and robustness. In the multidisciplinary sensitivity analysis of the coupled system, DYMORE5 operates in complex mode; the sensitivities of DYMORE5 solutions to aerodynamic load perturbations are assessed through complex-variable analysis.

A FUN3D/DYMORE5 interface has been developed to exchange aerodynamic loads and structural deflections between high-fidelity aero and structural dynamics models (Ref. 25). This interface has been extended to enable multidisciplinary sensitivity analysis that integrates adjoint-based CFD sensitivities and complex-variable CA sensitivities. The extended multidisciplinary system can conduct CFD/CA analysis based on loosely or tightly coupled approaches (Ref. 14).

\section{Mathematical Formulations}

A mathematical formulation for a tightly coupled multidisciplinary CFD/CA analysis is outlined in this section, followed by a formulation for sensitivity analysis of the coupled system. More detailed descriptions of the formulations can be found in Ref. 15.

In the present coupled system, a CA solution vector at time level $n$, denoted as $\mathbf{u}^{n}$, is provided at specified stations on the surface and used to update the CFD surface grid, $\mathbf{X}_{s}^{n}$, expressed as

$$
\mathbf{X}_{s}^{n}=\mathbf{T}_{s}^{n}\left(\mathbf{u}^{n}\right) \hat{\mathbf{X}}_{s}(\mathbf{D})
$$

where $\mathbf{T}_{s}^{n}$ denotes a transform matrix for elastic surface motion at time step $n$. The matrix varies at individual surface nodes and depends on the DYMORE5 solutions, $\mathbf{u}^{n}$. The reference surface grid, $\hat{\mathbf{X}}_{s}$, is obtained based on a surface parameterization and explicitly depends on the design (shape) parameters, D. The reference surface grid is not affected by elastic deformations or rigid body motions. A vector of CFD solutions, $\mathbf{Q}^{n}$, is computed on the overset volume grid, $\mathbf{X}^{n}$, at time level $n$. A vector of airloads, $\mathbf{f}^{n}$, is computed by the CFD code at time level $n$ at the airstations and transferred to the CA code to compute structural responses. The following equations for a tight-coupling formulation are written in the order in which the equations are solved. Here, the BDF1 scheme is used for simplification of the presentation.

$$
\begin{array}{cc}
\text { Reference Grid : } \hat{\mathbf{G}}(\hat{\mathbf{X}}, \hat{\mathbf{X}} s, \mathbf{D}) & =\mathbf{0}, \\
\text { Initial Grid : } \mathbf{G}^{0}\left(\mathbf{X}^{0}, \hat{\mathbf{X}}, \mathbf{T}_{r}^{0}\left(\mathbf{u}^{0}\right), \mathbf{D}\right) & =\mathbf{0}, \\
\text { Initial Flow : } \mathbf{R}^{0}\left(\mathbf{Q}^{0}, \overline{\mathbf{Q}}(\mathbf{D})\right) & =\mathbf{0}, \\
\text { Initial Load : } \mathbf{F}^{0}\left(\mathbf{f}^{0}, \mathbf{Q}^{0}, \mathbf{X}^{0}\right) & =\mathbf{0}, \\
\text { CA : } \mathbf{C}^{n}\left(\mathbf{u}^{n}, \mathbf{u}^{n-1}, \mathbf{f}^{n-1}, \mathbf{D}\right) & =\mathbf{0},
\end{array}
$$




$$
\begin{aligned}
\text { CFD Grid : } \mathbf{G}^{n}\left(\mathbf{X}^{n}, \mathbf{T}_{r}^{n}\left(\mathbf{u}^{n}\right), \mathbf{X}_{s}^{n}\left(\mathbf{u}^{n}\right), \hat{\mathbf{X}}, \mathbf{D}\right) & =\mathbf{0}, \\
\text { CFD Flow : } \mathbf{R}^{n}\left(\mathbf{Q}^{n}, \mathbf{Q}^{n-1}, \mathbf{X}^{n}, \mathbf{X}^{n-1}, \mathbf{D}\right) & =\mathbf{0}, \\
\text { Loads : } \mathbf{F}^{n}\left(\mathbf{f}^{n}, \mathbf{Q}^{n}, \mathbf{X}^{n}\right) & =\mathbf{0},
\end{aligned}
$$

where $\hat{\mathbf{X}}$ represents the reference volume grid obtained by solving the linear elasticity equations to transmit the surface mesh displacements throughout the interior field; $\mathbf{T}_{r}^{n}$ is a transform matrix evaluated by an averaged rigid motion extracted from DYMORE5 solutions, $\mathbf{u}^{n} ; \overline{\mathbf{Q}}$ represents a given initial flow condition; and the superscript index $n$ denotes the time step in the range of $1 \leq n \leq N$, where $N$ is the total number of time steps.

In the sensitivity analysis framework presented here, the CFD flow and grid equations and the loads equations use adjoint formulations, whereas sensitivities of the surface grid and the extracted rigid motion to the aerodynamic loads are computed by a complex-variable perturbation method (Ref. 13). For a general objective function $f(\mathbf{Q}, \mathbf{u}, \mathbf{X}, \mathbf{f}, \mathbf{D})$, the adjoint formulation is derived by differentiating a Lagrangian functional, $L$, formed as

$$
\begin{aligned}
L=f(\mathbf{Q}, \mathbf{u}, \mathbf{X}, \mathbf{f}, \mathbf{D})+ & \sum_{n=0}^{N}\left[\boldsymbol{\Lambda}_{R}^{n}\right]^{T} \mathbf{R}^{n}+\sum_{n=0}^{N}\left[\boldsymbol{\Lambda}_{G}^{n}\right]^{T} \mathbf{G}^{n} \\
& +\sum_{n=0}^{N}\left[\boldsymbol{\Lambda}_{F}^{n}\right]^{T} \mathbf{F}^{n}+\left[\boldsymbol{\Lambda}_{\hat{G}}\right]^{T} \hat{\mathbf{G}}
\end{aligned}
$$

where $\mathbf{Q}, \mathbf{u}, \mathbf{X}$, and $\mathbf{f}$ represent CFD solutions, CA surface displacements, grids, and airloads solutions at all time levels, respectively; $\boldsymbol{\Lambda}_{R}, \boldsymbol{\Lambda}_{G}$, and $\boldsymbol{\Lambda}_{F}$ are vectors of the time-dependent adjoint solutions for the flow, grid, and load equations, respectively; $\boldsymbol{\Lambda}_{\hat{G}}$ denotes the adjoint solution for the reference grid; and the superscript $T$ represents the transposition operator. Differentiating the Lagrangian with respect to design parameters $\mathbf{D}$ and equating the coefficients of state-variable sensitivities to zero (Ref. 26) yields the following adjoint equations:

$$
\begin{gathered}
\text { Load : }\left[\frac{\partial f}{\partial \mathbf{f}^{n}}\right]+\left[\boldsymbol{\Lambda}_{F}^{n}\right]^{T}\left[\frac{\partial \mathbf{F}^{n}}{\partial \mathbf{f}^{n}}\right]+\sum_{k=n+1}^{N}\left[\boldsymbol{\Lambda}_{G}^{k}\right]^{T}\left[\frac{\partial \mathbf{G}^{k}}{\partial \mathbf{f}^{n}}\right]=\mathbf{0}, \\
\text { Flow : }\left[\frac{\partial f}{\partial \mathbf{Q}^{n}}\right]+\sum_{j=n}^{n+1}\left[\boldsymbol{\Lambda}_{R}^{j}\right]^{T}\left[\frac{\partial \mathbf{R}^{j}}{\partial \mathbf{Q}^{n}}\right]+\left[\boldsymbol{\Lambda}_{F}^{n}\right]^{T}\left[\frac{\partial \mathbf{F}^{n}}{\partial \mathbf{Q}^{n}}\right]=\mathbf{0}, \\
\text { Grid : }\left[\frac{\partial f}{\partial \mathbf{X}^{n}}\right]+\left[\boldsymbol{\Lambda}_{G}^{n}\right]^{T}\left[\frac{\partial \mathbf{G}^{n}}{\partial \mathbf{X}^{n}}\right]+\sum_{j=n}^{n+1}\left[\boldsymbol{\Lambda}_{R}^{j}\right]^{T}\left[\frac{\partial \mathbf{R}^{j}}{\partial \mathbf{X}^{n}}\right] \\
+\left[\boldsymbol{\Lambda}_{F}^{n}\right]^{T}\left[\frac{\partial \mathbf{F}^{n}}{\partial \mathbf{X}^{n}}\right]=\mathbf{0}, \\
\text { Reference Grid : }\left[\boldsymbol{\Lambda}_{\hat{G}}\right]^{T}\left[\frac{\partial \hat{\mathbf{G}}}{\partial \hat{\mathbf{X}}}\right]+\sum_{m=0}^{N}\left[\boldsymbol{\Lambda}_{G}^{m}\right]^{T}\left[\frac{\partial \mathbf{G}^{m}}{\partial \hat{\mathbf{X}}}\right]=\mathbf{0},
\end{gathered}
$$

where the time step $n$ ranges from $N$ to 0 , and thus, the multidisciplinary adjoint solutions are solved in the reverse order in time. In the current implementation, $\left[\partial \mathbf{F}^{n} / \partial \mathbf{f}^{n}\right]$ in Eq. (11) is the identity matrix. The term $\left[\partial \mathbf{G}^{k} / \partial \mathbf{f}^{n}\right]$ in this equation denotes sensitivities of the CFD grid at time level $k$ to aerodynamic loads at time level $n$. Specifically, this sensitivity term is computed using the chain rule as

$$
\left[\frac{\partial \mathbf{G}^{k}}{\partial \mathbf{f}^{n}}\right]=\left\{\left[\frac{\partial \mathbf{G}^{k}}{\partial \mathbf{T}_{r}^{k}}\right]\left[\frac{\partial \mathbf{T}_{r}^{k}}{\partial \mathbf{u}^{k}}\right]+\left[\frac{\partial \mathbf{G}^{k}}{\partial \mathbf{X}_{s}^{k}}\right]\left[\frac{\partial \mathbf{X}_{s}^{k}}{\partial \mathbf{u}^{k}}\right]\right\}\left[\frac{\partial \mathbf{u}^{k}}{\partial \mathbf{f}^{n}}\right],
$$

where $\left[\partial \mathbf{u}^{k} / \partial \mathbf{f}^{n}\right]$ denotes sensitivities of DYMORE5 solutions at time level $k$ to aerodynamic loads at time level $n$. The terms $\left[\partial \mathbf{G}^{k} / \partial \mathbf{T}_{r}^{k}\right]$ and $\left[\partial \mathbf{G}^{k} / \partial \mathbf{X}_{s}^{k}\right]$ are manually differentiated. The sensitivities $\left[\partial \mathbf{T}_{r}^{k} / \partial \mathbf{u}^{k}\right],\left[\partial \mathbf{X}_{s}^{k} / \partial \mathbf{u}^{k}\right]$, and $\left[\partial \mathbf{u}^{k} / \partial \mathbf{f}^{n}\right]$ are assessed by complex-variable perturbation computations. To assess $\left[\partial \mathbf{u}^{k} / \partial \mathbf{f}^{n}\right]$, an airload component at time level $n$ is perturbed by $i 10^{-50}$, and the complex-variable DYMORE5 solutions $\mathbf{u}^{k}$ are computed for subsequent time levels $k, n+1 \leq k \leq N$. The real part of $\mathbf{u}^{k}$ is identical to that computed in the forward CFD/CA analysis, while the imaginary part (divided by $10^{-50}$ ) is the sensitivity of $\mathbf{u}^{k}$ to the particular airload component. Since the airload perturbations for individual components are completely independent operations, the assessment of sensitivities of DYMORE5 solutions to aerodynamic loads is performed in parallel. The sensitivities of rigid and surface elastic motions to DYMORE solutions, $\left[\partial \mathbf{T}_{r}^{k} / \partial \mathbf{u}^{k}\right]$ and $\left[\partial \mathbf{X}_{s}^{k} / \partial \mathbf{u}^{k}\right]$ in Eq. (15), are computed in a similar procedure. Each component of the DYMORE5 solutions is perturbed with $i 10^{-50}$ to assess the corresponding motion sensitivity. These computations are confined to time level $k$ and also performed in parallel.

From Eq. (11), the collective time of evaluating sensitivities of all CFD grids to all airload components is quadratically proportional to the number of time steps, linearly proportional to the number of airload components (or, equivalently, DYMORE5 solution components) at a time level, and inversely proportional to the number of processors used in the complexvariable sensitivity analysis. In the present work, the number of processing cores is selected to be the same as the number of airload components at a time level. This selection removes the linear dependence of the collective time on the number of airload components.

Equations (11)-(13) are subject to the following conditions: $\boldsymbol{\Lambda}_{G}^{N+1}=0$ and $\boldsymbol{\Lambda}_{R}^{N+1}=0$. The final multidisciplinary sensitivity derivatives are computed as

$$
\begin{aligned}
\frac{d L}{d \mathbf{D}} & =\left[\frac{\partial f}{\partial \mathbf{D}}\right]+\sum_{n=0}^{N}\left[\boldsymbol{\Lambda}_{R}^{n}\right]^{T}\left[\frac{\partial \mathbf{R}^{n}}{\partial \mathbf{D}}\right]+\left[\boldsymbol{\Lambda}_{\hat{G}}\right]^{T}\left[\frac{\partial \hat{\mathbf{G}}}{\partial \mathbf{D}}\right] \\
& +\sum_{n=0}^{N}\left[\boldsymbol{\Lambda}_{G}^{n}\right]^{T}\left[\frac{\partial \mathbf{G}^{n}}{\partial \mathbf{D}}\right] .
\end{aligned}
$$

Verification of this sensitivity analysis framework is provided in Ref. 15.

\section{Design Optimization Cycle}

A sequential quadratic-programming optimizer, SNOPT (Refs. 27, 28), is used to drive gradient-based rotorcraft design optimization. The design optimization cycle is illustrated in Fig. 1 and summarized as the following steps. 


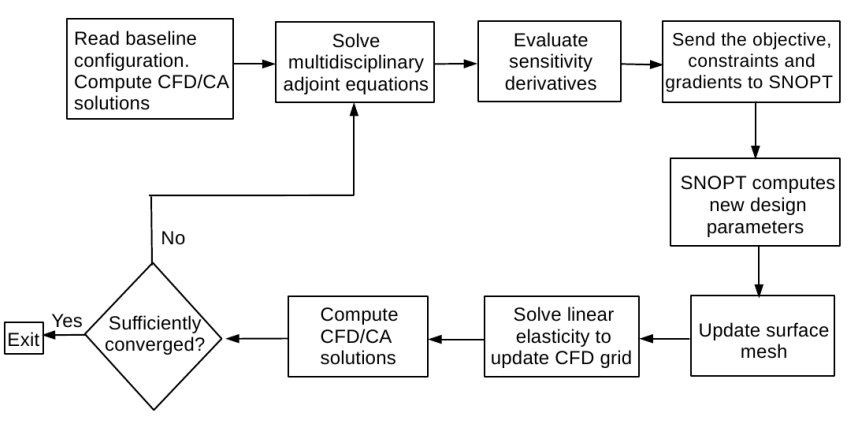

Fig. 1. Flowchart illustrating design optimization cycle.

1. The cycle starts from the baseline configuration and initial design parameters. Multidisciplinary CFD/CA solutions are computed. The values of the objective function and constraints are evaluated.

2. The multidisciplinary adjoint equations are solved by marching in reverse time. The latest available design parameters and the corresponding CFD flow, CFD grid, and CA solutions are used.

3. The sensitivities of the objective and constraints to desired design parameters are evaluated.

4. The optimizer is provided with the current values of the objective and constraints, the current values of the design parameters, the sensitivities of the objective and constraints to the design parameters, as well as lower and upper bounds of the design parameters.

5. SNOPT provides corrections for the design parameters. Those corrections are applied to compute new design parameters.

6. The surface grid is updated using new design parameters.

7. The CFD volume grid is updated by using a linear elasticity approach.

8. The tight-coupling CFD/CA forward analysis is performed with the new design parameters. The values of the objective function and constraints are evaluated.

9. Steps of 2-8 are repeated until the objective function is sufficiently minimized, and constraints are converged to a satisfactory level.

\section{NUMERICAL RESULTS}

In this section, a verification study is first conducted to confirm accuracy of the coupled CFD/CA solution that employs the new DYMORE5 model. Next, a constrained gradientbased design optimization is demonstrated for a HART-II rotorcraft configuration in a descending flight condition.

\section{CFD/CA Loose-Coupling Analysis}

The benchmark HART-II baseline test model (Ref. 7) is used for verification of coupling of the FUN3D solver with the new
DYMORE5 model. In the previous work (Ref. 14), a similar study had been performed to verify coupling with the older DYMORE4 model. The flow conditions in the HART-II baseline case include a tip Mach number of 0.6387, an advance ratio of 0.151 , and a shaft tilt angle of $4.5^{\circ}$. This case represents descending flight, where blade-vortex interactions are significant. Other important parameters of the blade geometry and test conditions are detailed elsewhere (Ref. 7).

Loose-coupling simulations for the HART-II baseline case conducted with the current system are compared to FUN3D/CAMRAD simulations (Ref. 5), FUN3D/DYMORE4 simulations (Ref. 14), and experimental data. Although the structural model parameters used in the CAMRAD and DYMORE solvers are similar, small differences in common parameters are observed. In addition, each CA solver has specific input parameters, which can lead to slightly different structural dynamics modeling. The unstructured, mixed-element, overset grid used in the present CFD simulation consists of four blade component grids, one fuselage component grid, and a stationary background grid, resulting in a total of 7,058,618 nodes and 29,030,886 elements $(22,799,206$ tetrahedrons, 44,874 pyramids, and $6,186,806$ prisms). The BDF2opt temporal scheme is used to advance in time with a time step corresponding to the increment of $1^{\circ}$ azimuth angle. At the end of 12 coupling cycles that involve 6 revolutions, variation of the control angles and thrust deltas between coupling cycles is less than $0.001^{\circ}$ and $0.01 \%$, respectively. Tables 1 and 2 summarize the loose-coupling solutions for various aerodynamic metrics and the trim control angles obtained by the FUN3D and DYMORE5 solvers.

Figures 2(a)-2(b) compare the loose-coupling predictions of the normal force and pitching moment coefficients at $87 \%$ blade radial station with the measurement data. The current FUN3D/DYMORE5 and previous FUN3D/DYMORE4 simulations are close, where the root-mean-square (RMS) norm of difference for the normal-force and pitching-moment coefficients is 0.0007 and 0.00003 , respectively. The magnitudes and shapes of the normal-force and pitching-moment profiles predicted by the current solutions are in good agree-

Table 1. Thrust, rolling and pitching moment coefficients $\left(C_{T}, C_{M x}\right.$, and $\left.C_{M y}\right)$ of FUN3D/DYMORE5 loose-coupling analysis.

\begin{tabular}{cccc}
\hline Output & $C_{T}$ & $C_{M x}$ & $C_{M y}$ \\
\hline Targets & $4.57 \times 10^{-3}$ & $1.39 \times 10^{-5}$ & $-1.39 \times 10^{-5}$ \\
FUN3D & $4.61 \times 10^{-3}$ & $1.46 \times 10^{-5}$ & $-1.14 \times 10^{-5}$ \\
DYMORE5 & $4.58 \times 10^{-3}$ & $1.54 \times 10^{-5}$ & $-1.50 \times 10^{-5}$ \\
\hline
\end{tabular}

Table 2. Trim control angles of FUN3D/DYMORE5 loosecoupling analysis.

\begin{tabular}{cccc}
\hline Output & $\theta_{0}$ & $\theta_{1 c}$ & $\theta_{1 s}$ \\
\hline Experiment & $3.8^{\circ}$ & $1.92^{\circ}$ & $-1.34^{\circ}$ \\
FUN3D/DYMORE5 & $4.0^{\circ}$ & $1.86^{\circ}$ & $-1.12^{\circ}$ \\
\hline
\end{tabular}


ment with those of the FUN3D/CAMRAD solutions. The maximum difference in the normal-force coefficient between the FUN3D/DYMORE5 and FUN3D/CAMRAD analyses is observed close to $114^{\circ}$ and does not exceed 0.004. The maximum difference in the pitching-moment coefficient between the current and FUN3D/CAMRAD analyses is observed close to $126^{\circ}$ and does not exceed 0.0003. Compared to the experimental measurement, the blade-vortex interactions (BVI), particularly at the retreating side, are captured reasonably well by the FUN3D/DYMORE5 analysis. The

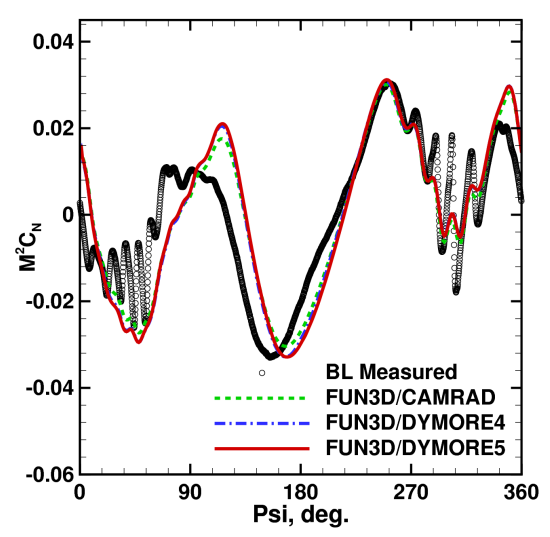

(a) Normal force

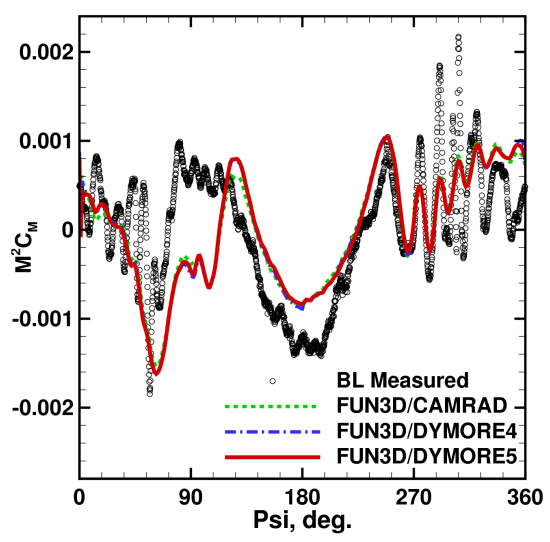

(b) Pitching moment

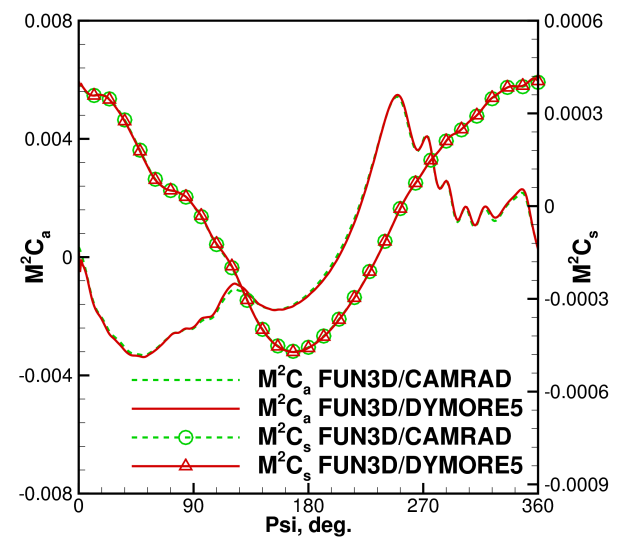

(c) Chord and spanwise forces

Fig. 2. Comparisons of force and moment predictions at $87 \%$ radial station with means removed. accuracy for capturing the BVI events is improved with increased grid resolution (Ref. 14). Figure 2(c) shows comparisons of the chord- and spanwise forces at this blade radial station. Though experimental data is not available for these forces, the FUN3D/DYMORE5 analysis exhibits consistently good agreement with the forces obtained from the FUN3D/CAMRAD analysis.

Figure 3 compares the azimuthal variations of elastic motions including flap, lead-lag and torsion deformations at the blade tip. The flap deflections are obtained by removing the

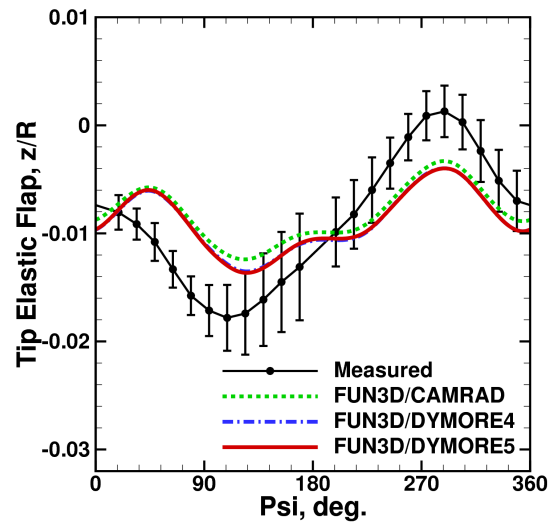

(a) Flap relative to precone

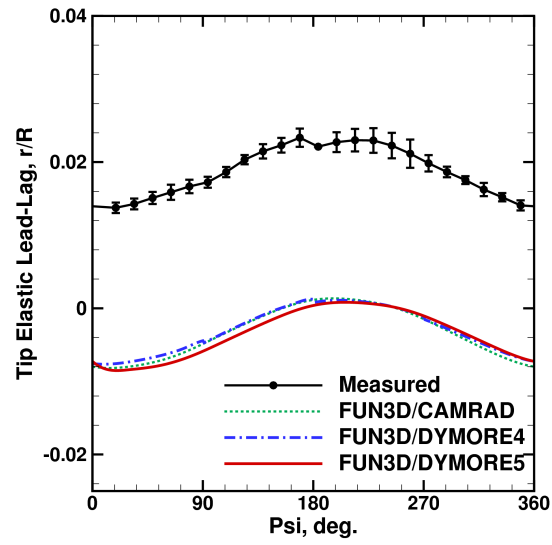

(b) Lead-lag

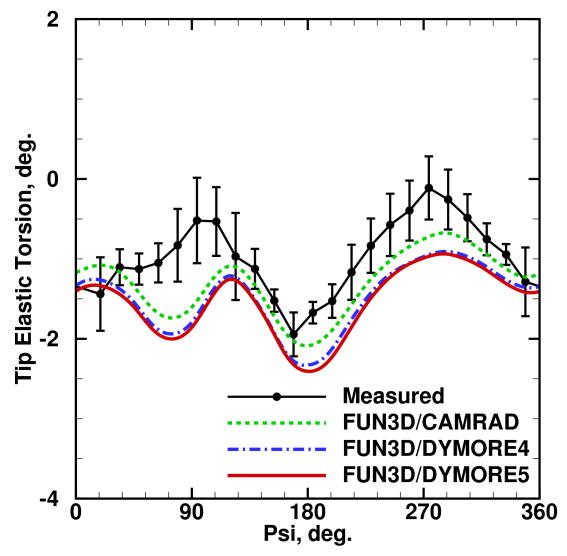

(c) Torsion relative to pretwist and control angles

Fig. 3. Blade tip flap, lead-lag and torsion deflections. 
precone angle $\left(2.5^{\circ}\right)$ from the vertical displacements, while the elastic torsion is obtained by excluding the pretwist angle and the pitch control inputs from the total geometric pitch angle. The magnitude and phase of the predicted blade tip flap deflections in the FUN3D/DYMORE5 solution agree well with those of the FUN3D/CAMRAD solution. Compared to the measurement data, a constant offset roughly amounting to $1 / 5$ of the blade chord in the elastic lead-lag deflections is observed; this translational offset behavior is commonly seen in other work (Refs. 29,30). In comparison to the previous simulation involving the DYMORE4 model, the FUN3D/DYMORE5 solutions show generally good agreement; only a small phase shift (approximately 6 deg.) is observed in the tip lead-lag deflections. Further investigation will be performed to explain this small difference. The tip elastic torsion is in a good agreement between the results involving the two DYMORE models, where the RMS norm of the difference is $0.06^{\circ}$; and the $2 / \mathrm{rev}$ behavior is observed. In addition, the FUN3D/DYMORE5 solution shows approximately $0.4^{\circ}$ underpredictions from the FUN3D/CAMRAD solution across all azimuth locations. This nearly constant offset is likely due to a small difference in the control angles predicted by the DYMORE5 and CAMRAD comprehensive analysis models.

\section{Rotorcraft Design Optimization}

In this section, the multidisciplinary sensitivity analysis is applied to gradient-based design optimization of the baseline HART-II rotorcraft configuration. The computational grid used in the preceding section is also used for this optimization demonstration. The BDF2opt time integration scheme is used in FUN3D, and the time step corresponds to $1^{\circ}$ azimuth angle of rotor rotation. At each time step, 20 nonlinear subiterations are performed. The tolerance for the linear elasticity solver is set to $10^{-10}$. To avoid nonphysical deflections of the blades and alleviate the initial transient effects, FUN3D and DYMORE5 are initialized with the loose-coupling CFD/CA solutions. The same initial conditions are used in every design cycle during the optimization process.

The objective for the optimization demonstration is to reduce the time-averaged rotor torque coefficient while explicitly constraining the thrust and the rolling and pitching moments to the target values corresponding to the baseline rotor performance (see Table 1). Due to the relation between the rotor shaft power and torque, $P=\Omega Q(P, Q$ and $\Omega$ denote the shaft power, torque, and angular speed of the rotor, respectively), the current objective can also be used for minimization of the rotor power. The objective and constraints are expressed as the following time-averaged functions:

$$
\begin{gathered}
f_{o b j}=w_{o b j}\left[\left(\frac{1}{N_{2}-N_{1}+1} \sum_{n=N_{1}}^{N_{2}} C_{Q}^{n}\right)-C_{Q}^{*}\right]^{2} \Delta t, \\
g_{1}=w_{1}\left[\left(\frac{1}{N_{2}-N_{1}+1} \sum_{n=N_{1}}^{N_{2}} C_{T}^{n}\right)-C_{T}^{*}\right]^{2} \Delta t,
\end{gathered}
$$

$$
\begin{aligned}
& g_{2}=w_{2}\left[\left(\frac{1}{N_{2}-N_{1}+1} \sum_{n=N_{1}}^{N_{2}} C_{M x}^{n}\right)-C_{M x}^{*}\right]^{2} \Delta t, \\
& g_{3}=w_{3}\left[\left(\frac{1}{N_{2}-N_{1}+1} \sum_{n=N_{1}}^{N_{2}} C_{M y}^{n}\right)-C_{M y}^{*}\right]^{2} \Delta t .
\end{aligned}
$$

For rotor torque minimization, $C_{Q}^{*}$ is set to zero; $C_{T}^{*}, C_{M x}^{*}$, and $C_{M y}^{*}$ are target values of thrust, and rolling and pitching moments, respectively, set as $C_{T}^{*}=0.00457, C_{M x}^{*}=0.0000139$, and $C_{M y}^{*}=-0.0000139$ based on the dimensional quantities of the baseline configuration. The term $\Delta t$ is the nondimensional time-step size. The objective and constraints given in Equations (17)-(20) are computed from forces and moments on the rotor blade surfaces; the forces and moments on the fuselage surface have been excluded. Weighting parameters, $w_{o b j}, w_{1}, w_{2}$, and $w_{3}$, provide scaling of the objective and constraints. These weighting parameters are based on the values of the objective and constraints corresponding to the baseline configuration and/or the norms of sensitivity derivatives of the output functions. Parameters $N_{1}$ and $N_{2}$ represent the first and last time-level indices of the optimization interval. They are set as $N_{1}=271$ and $N_{2}=360$, respectively. Therefore, the optimization interval is the fourth quarter of the time period corresponding to the first rotor revolution.

In the current study, a geometry parameterization tool, Multidisciplinary Aerodynamic-Structural Shape Optimization Using Deformation (MASSOUD) software package (Ref. 31) is employed for surface grid deformation. The parameterization tool uses a set of aircraft-centric shape design parameters such as planform, twist, shear, thickness, and camber to realize general configuration shape control. In the present optimization demonstration, the set of design parameters consists of 79 shape parameters on each of the four HART-II rotor blades, including 8 twist parameters, 35 thickness parameters, and 36 camber parameters. The blade planform geometry of the HART-II rotor and locations of twist, thickness, and camber shape design parameters are illustrated in Fig. 4. The thickness of leading and trailing edges is not allowed to be modified. Camber variables are not allowed to be adjusted at the trailing edge nor in the blade root region. In addition, three control angles describing the collective and cyclic control inputs are used as design parameters and are allowed to vary as much as \pm 5 degrees. The initial inputs of control angles for the baseline configuration are taken as the trim control angles computed by the looselycoupled FUN3D/DYMORE5 system. The quasisteady trimmer in the DYMORE5 model is not involved in the rotorcraft design optimization; the trim conditions are enforced by

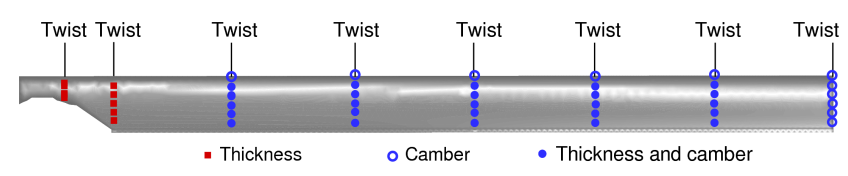

Fig. 4. Planform geometry and locations of shape design parameters on HART-II rotor blade. 
SNOPT through constraining the thrust, rolling, and pitching moments. One distinction between the trim processes of DYMORE5 and SNOPT is that DYMORE5 performs trimming based on the sectional forces and moments evaluated at airstations, whereas the SNOPT optimizer uses airloads evaluated from the entire surface grid.

\section{Optimization Results}

The optimization has been performed on the NASA Advanced Supercomputing (NAS) facility at the Ames Research Center, with the execution conducted on 2088 processing cores. In this computation environment, executions of the coupled CFD/CA and multidisciplinary sensitivity analyses within a single optimization cycle require 2.0 and 6.2 hours of wallclock time, respectively. Detailed analysis of the computational cost of individual components is provided in the following section.

Figures 5 and 6 show convergence of the torque coefficient and constraints. A $3.23 \%$ reduction in the rotor torque coefficient is achieved after 13 design cycles. Due to the fact that a loose-coupling solution is used as the initial condition for the tight-coupling analysis, all constraints for the baseline configuration are close to the target values, as shown in Fig. 6(a). Specifically, the thrust, and rolling and pitching moment coefficients computed for the baseline configuration are $4.61 \times 10^{-3}, 1.41 \times 10^{-5}$, and $-1.10 \times 10^{-5}$, respectively. These aerodynamic metrics are computed from the first rotor revolution of the tight-coupling procedure and agree well with the loose-coupling solutions and targets shown in Table 1 . In addition, the thrust and rolling and pitching moment constraints of the optimized tight-coupling solutions further converge to the target values and provide better matches than the loose-coupling solutions of the baseline configuration. Table 3 lists the absolute and relative differences of the constraints from the target values. Specifically, the pitching moment coefficient is within $0.07 \%$ of the target value; all other constraints are well within $0.03 \%$ of the target values.

Figure 7 depicts the convergence history of the collective and cyclic pitch control angles. Variation of the control

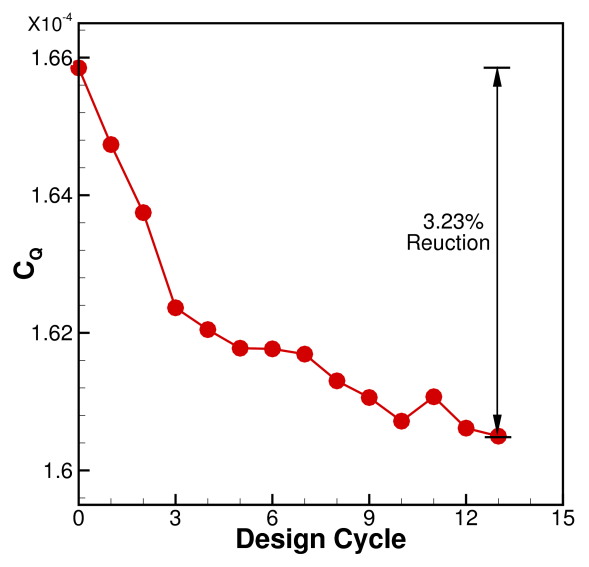

Fig. 5. Convergence of rotor torque.

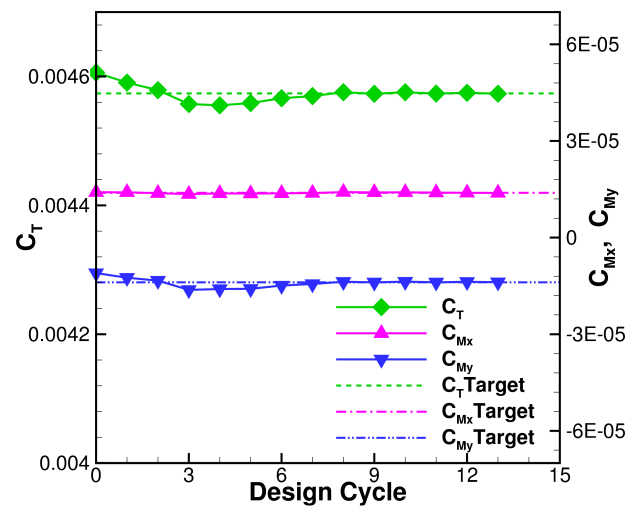

Fig. 6. Convergence of thrust and moments.

angles from the (initial) values corresponding to the loosecoupling simulation shown in Table 2 is less than $0.6^{\circ}$ during the entire optimization process. The converged collective, lateral cyclic and longitudinal cyclic control angles for the optimized HART-II rotorcraft configuration are $3.69^{\circ}, 1.68^{\circ}$, and $-1.17^{\circ}$, respectively. Compared to the trim control angles obtained from the loose-coupling solution, the collective control angle decreases by $0.31^{\circ}$, adjusting the thrust coefficient back to the target; the lateral and longitudinal cyclic control angles decrease by $0.18^{\circ}$ and $0.05^{\circ}$, respectively. Overall, the computed pitch controls remain close to the loose-coupling and experimental results.

Figure 8(a) displays a snapshot of the elastic blades after one revolution for the baseline and optimized configurations. The blades corresponding to the optimized geometry are colored with the surface pressure coefficient levels and shown on the top; the baseline blades are shaded and shown at the bot-

Table 3. Deviation of thrust and rolling and pitching moments of optimized configuration from targets.

\begin{tabular}{cccc}
\hline \multirow{2}{*}{ Constraint } & Target & $\begin{array}{c}\text { Absolute } \\
\text { Difference }\end{array}$ & $\begin{array}{c}\text { Relative } \\
\text { Difference }\end{array}$ \\
\hline$C_{T}$ & $4.57 \times 10^{-3}$ & $3.9 \times 10^{-7}$ & $0.009 \%$ \\
$C_{M x}$ & $1.39 \times 10^{-5}$ & $4.7 \times 10^{-9}$ & $0.03 \%$ \\
$C_{M y}$ & $-1.39 \times 10^{-5}$ & $9.7 \times 10^{-9}$ & $0.07 \%$ \\
\hline
\end{tabular}

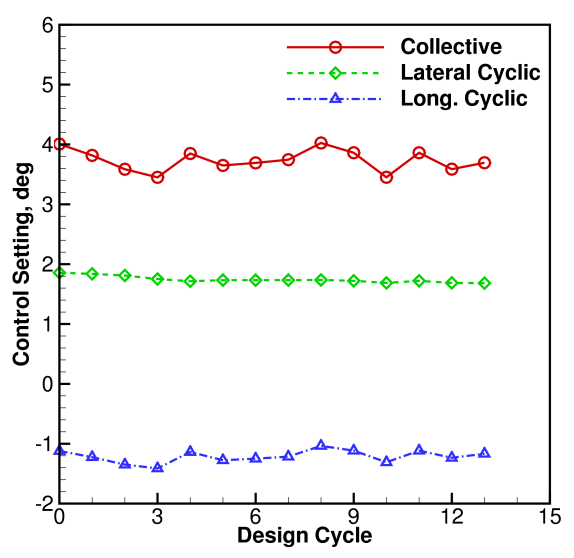

Fig. 7. Variation of pitch control angles. 
tom for comparison. The change in the optimized and baseline blade deflections is almost indistinguishable. Note that the constraints of the optimization problem penalize changes in the trim parameters. Fig. 8(b) compares the elastic flap deflections at the blade tip for the tight-coupling solutions corresponding to the optimized and baseline configurations and the loose-coupling solution shown in the preceding section. The tight-coupling solution of the baseline configuration is almost identical with the loose-coupling solution, indicating that the tight-coupling algorithm of the FUN3D/DYMORE5 system functions correctly. The amplitude of the flap deflections produced by the optimized configuration is reduced compared to the baseline configuration, especially in the second and third quadrants; the maximum reduction of approximately 0.0025 of the blade radius occurs near the $185^{\circ}$ and $272^{\circ}$ azimuths.

Airfoil cross-sections of the baseline and optimized blade geometries are shown in Fig. 9 for spanwise stations ranging from $25 \%$ to $98 \%$ of the blade radius; an enlarged vertical scale is used to view details. The observed changes in the airfoil cross-sections are results of combined changes in many shape design parameters. In comparison to the baseline geometry, the optimized shape has more negative twist (i.e., nose down) across most of the blade span. Reduced thickness

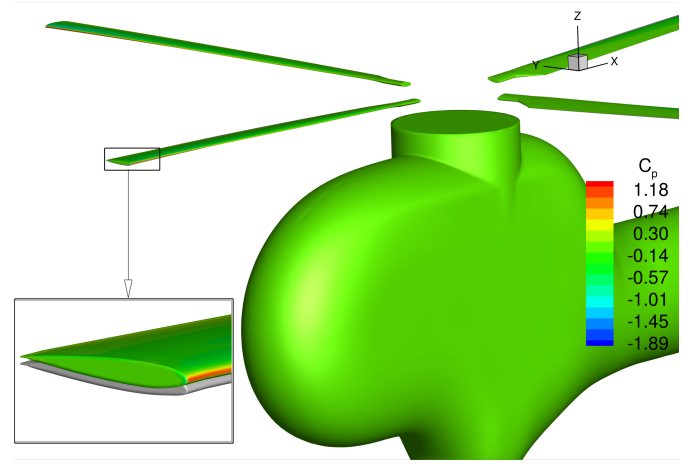

(a) Overview

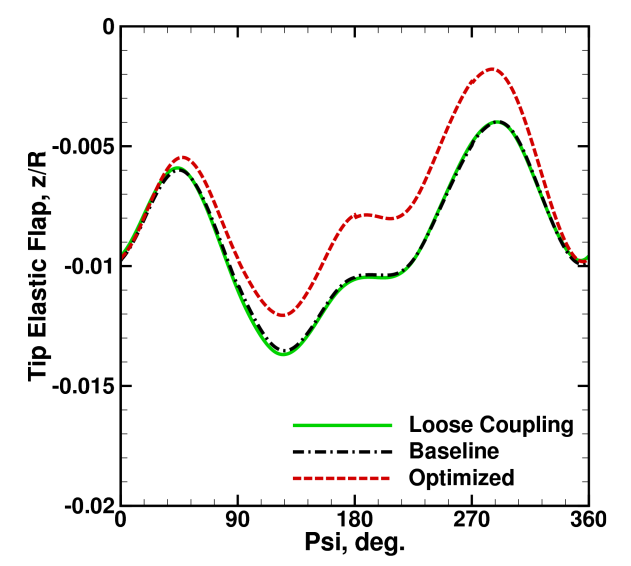

(b) Elastic flap at blade tip relative to precone

Fig. 8. Blade deflections of the baseline and optimized configurations (baseline shown at bottom and optimized on top).
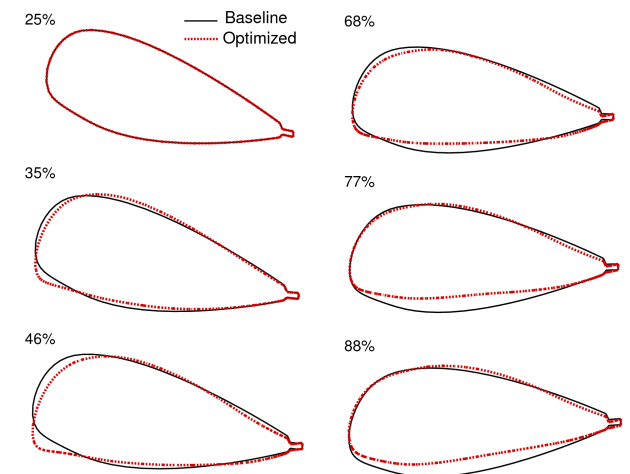

$77 \%$

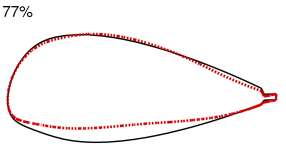

$88 \%$
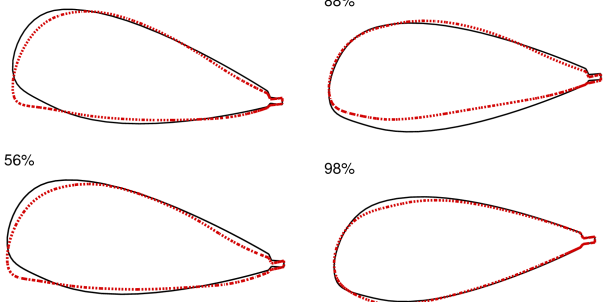

$98 \%$

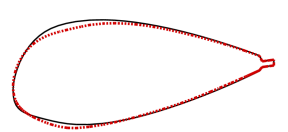

Fig. 9. Baseline and optimized blade section geometries in exaggerated vertical scales $(y: z=1: 3)$.

is clearly observed in outboard and aft regions. Negative camber is observed in aft regions close to the trailing edge, while increased positive camber is observed in front and midchord regions. In addition, the lower surface of the airfoil appears more flat, particularly in the middle of the blade span. This change in airfoil geometry leads to higher lift to compensate for the adverse effects from the lower twist angles and collective pitch control angle.

Figure 10 shows time histories of the torque, thrust, and rolling and pitching moments for the baseline and optimized configurations, obtained from 10 revolutions of tight-coupling simulations based on the FUN3D/DYMORE5 system. These long-term simulations assess aerodynamic metrics in the limit of a periodic solution for the optimized configuration. Although the optimization interval is set within a short period of simulation ( $1 / 4$ revolution), the reduction of rotor torque is well maintained beyond the optimization time interval. The mean values of thrust, rolling and pitching moments are preserved, indicating that the required trim conditions have been maintained.

\section{Performance of Design Optimization Cycle}

For the design optimization problem presented in the preceding sections, a breakdown of the time usage within a design cycle is provided in Table 4. The total wall-clock times used to solve the tightly coupled CFD/CA analysis equations, the load adjoint equations, the flow adjoint equations, and the grid adjoint equations are shown separately in the table as well as the percentage of time spent on solutions of particular sets of equations. The total time for computing adjoint solutions is a factor of 3.1 greater than the time for computing the CFD/CA analysis solution. One reason for the higher cost of the multidisciplinary sensitivity analysis is the need to compute four adjoint solutions corresponding to one objective and three constraints. The other reason is that the computational time required to compute complex-variable mesh sensitivities to airloads grows quadratically with the number of time steps 


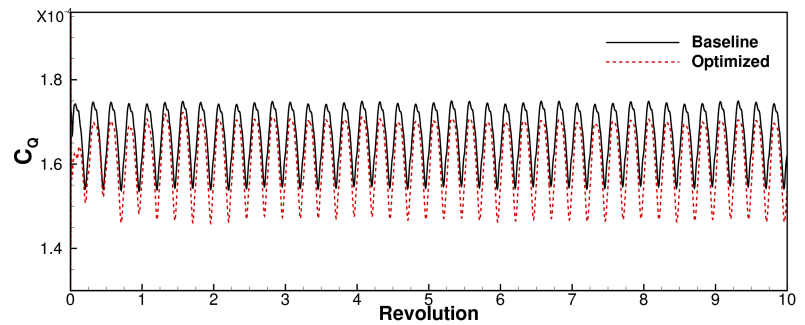

(a) Torque

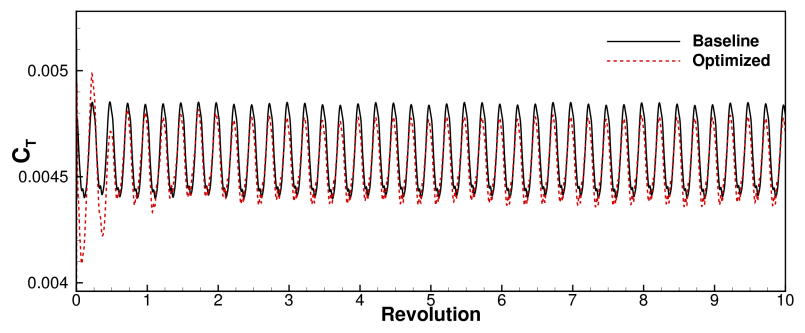

(b) Thrust

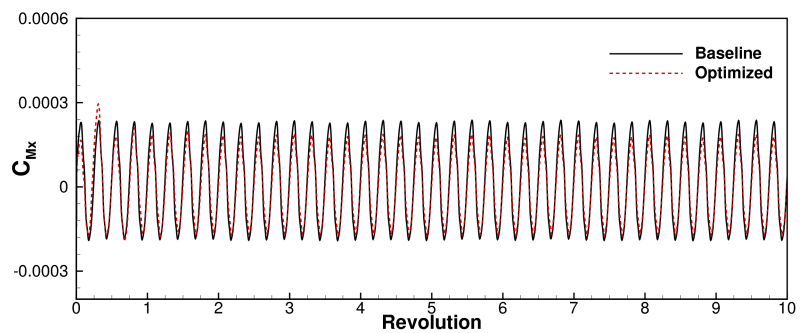

(c) Rolling moment

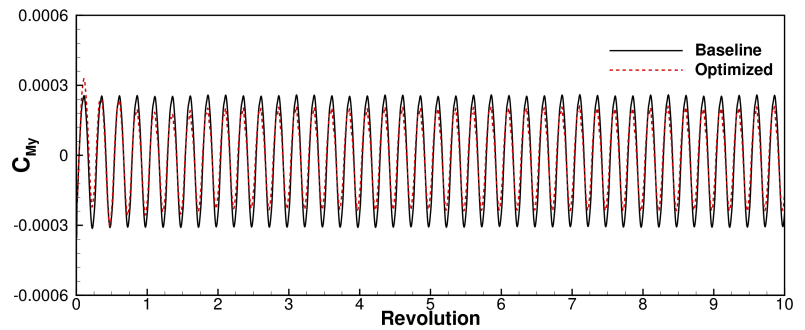

(d) Pitching moment

Fig. 10. Long-term tight-coupling simulations of baseline and optimized configurations.

(see Equations (11) through (15)). This quadratic dependence affects the time of computing load adjoint solutions.

In the previous study (Ref. 15), the time to compute a load adjoint solution with the same environment setting was about 13.7 hours (62\% of one design cycle time). The time for a load adjoint solution in the present study has been significantly reduced to 2.9 hours. This performance improvement is attributed to the following advancements. First, the new CA model, DYMORE5, replaces the former DYMORE4 model in the CFD/CA tight-coupling analysis. This new CA model eliminates excessive matrix factorization and leads to fewer iterations for computing DYMORE solutions at each time step. The DYMORE5 model reduces the DYMORE execution time by a factor of 8. Although DYMORE5 supports parallel computations, this capability is not currently used.
Table 4. Design cycle breakdown ( 2088 processors).

\begin{tabular}{ccc}
\hline Components & Wall-clock time $(h)$ & Percent $(\%)$ \\
\hline CFD/CA analysis & 2.0 & 24.4 \\
Load adjoint & 2.9 & 35.4 \\
Flow adjoint & 2.1 & 25.6 \\
Grid adjoint & 1.2 & 14.6 \\
\hline
\end{tabular}

Engaging DYMORE5 instead of DYMORE4 reduces the load adjoint execution time by about 1 hour. Second, the majority of the execution time was the computation of complexvariable sensitivities of the surface grid motions to perturbations of airloads. For each perturbation, the structural displacements were propagated to the entire surface grid. The number of operations required for this computation is large, proportional to the number of surface-grid degrees of freedom. In the new FUN3D/DYMORE5 system, the number of operations for computing elastic surface sensitivities has been dramatically reduced by separating elastic surface sensitivities to DYMORE solutions from DYMORE solution sensitivities to aerodynamic loads (See Eq. (15)). In this new formulation, the product $\left[\Lambda_{G}^{k}\right]^{T}\left[\partial \mathbf{G}^{k} / \partial \mathbf{X}_{s}^{k}\right]\left[\partial \mathbf{X}_{s}^{k} / \partial \mathbf{u}^{k}\right]$ is precomputed. The term $\left[\partial \mathbf{X}_{s}^{k} / \partial \mathbf{u}^{k}\right]$ is computed in parallel, using the complex-variable analysis. This approach effectively removes the term $\left[\partial \mathbf{X}_{s}^{k} / \partial \mathbf{u}^{k}\right]$ from the computations with a quadratic dependence on the number of time steps. This improvement leads to a reduction of about 9.8 hours in the wall-clock time required to compute the load adjoint solution.

With the combination of these two key advancements, the time to compute a load adjoint solution becomes comparable to the time of the flow adjoint solution. Overall, the wallclock time for one multidisciplinary sensitivity analysis cycle has been reduced by a factor of 3.2 compared to the wall-clock time used by the previous FUN3D/DYMORE4 system.

An approach to further accelerate multidisciplinary sensitivity analysis is to compute DYMORE sensitivities with realvalued finite differences. Real-valued computations are about three times faster than complex-valued computations. Although a noticeable efficiency improvement over the present multidisciplinary sensitivity analysis can still be achieved, the cost of the finite-difference sensitivity analysis unavoidably grows quadratically with the number of time steps. Complete elimination of this quadratic dependence requires a discretelyconsistent adjoint formulation for DYMORE sensitivities. A separate effort is currently underway to develop adjoint capabilities in DYMORE.

\section{CONCLUSIONS}

This paper has presented a multidisciplinary design and optimization framework for high-fidelity rotorcraft computations conducted by a tightly-coupled multidisciplinary system including a computational fluid dynamics code, FUN3D, and a rotorcraft comprehensive analysis code, DYMORE5. A formulation for the tight-coupling multidisciplinary sensitivity analysis has been developed and implemented. The formulation integrates the adjoint-based sensitivities of flow and grid 
solutions available in FUN3D with DYMORE5 sensitivities computed by a complex-variable perturbation approach.

A constrained gradient-based design optimization procedure has been formulated and successfully applied to optimization of the HART-II rotorcraft configuration. The demonstration comprised the objective of reducing rotor torque while maintaining baseline thrust, rolling and pitching moments. This demonstration confirms the feasibility and effectiveness of the current high-fidelity multidisciplinary sensitivity analysis for rotorcraft applications. The computational cost for the multidisciplinary sensitivity analysis has been reduced significantly by the present FUN3D/DYMORE5 system, in which the more efficient DYMORE5 model is employed in place of the former DYMORE4 model; and the sensitivities of elastic surface motion to DYMORE5 solutions are computed separately from the sensitivities of DYMORE5 solutions to aerodynamic loads. The latter step removes a major computational hurdle from the computations with a quadratic dependence on the number of time steps. The performance of the multidisciplinary sensitivity analysis has been significantly improved in comparison to the performance of FUN3D/DYMORE4 system. Completion of 13 high-fidelity multidisciplinary optimization cycles took about 4.5 days of computations involving 2088 processors. Since the present multidisciplinary sensitivity analysis still relies on finite-difference sensitivities for DYMORE solutions, complete elimination of the quadratic dependence requires a discretely-consistent adjoint formulation for DYMORE sensitivities.

Author contact: Li Wang, li.wang@ nianet.org; Boris Diskin, bdiskin@nianet.org; Robert T. Biedron, robert.t.biedron@nasa.gov; Eric J. Nielsen, eric.j.nielsen@nasa.gov; Valentin Sonneville, vspsonn@umd.edu; Olivier A. Bauchau, obauchau@umd.edu.

\section{ACKNOWLEDGMENTS}

The work was supported by the NASA Revolutionary Vertical Lift Technology Project contract NNL15AB93T. The authors would like to thank William Jones at NASA Langley Research Center for assistance with geometry parameterization and Prof. Marilyn Smith at Georgia Institute of Technology for providing the initial FUN3D/DYMORE interface as well as the DYMORE inputs for the HART-II configuration. The authors would also like to acknowledge the support of the HART-II partners from the U.S. Army Aero-Flight Dynamics Directorate (AFDD), German Aerospace Center (DLR), German-Dutch Windtunnel (DNW), and French Aeronautics and Space Research Center (ONERA). Resources supporting this work were provided by the NASA High-End Computing (HEC) Program through the NASA Advanced Supercomputing (NAS) Division at Ames Research Center.

\section{REFERENCES}

${ }^{1}$ H. Saberi, M. Khoshlahjeh, R. A. Ormiston, M. J. Rutkowski, Overview of RCAS and application to advanced rotorcraft problems, American Helicopter Society 4th Decennial Specialists Conference on Aeromechanics (Jan. 2004).

${ }^{2}$ W. Johnson, Rotorcraft Aeromechanics, Cambridge Aerospace Series, Cambridge University Press, 2013.

${ }^{3}$ O. A. Bauchau, C. L. Bottasso, Y. G. Nikishkov, Modeling rotorcraft dynamics with finite element multibody procedures, Mathematical and Computer Modeling 33 (2001) 1113-1137.

${ }^{4}$ M. Potsdam, H. Yeo, W. Johnson, Rotor airloads prediction using loose aerodynamic/structural coupling, 60th Annual American Helicopter Society Forum (2004).

${ }^{5}$ R. T. Biedron, E. M. Lee-Rausch, Rotor airloads prediction using unstructured meshes and loose CFD/CSD coupling, AIAA Paper 2008-7341 (June 2008).

${ }^{6}$ J. Sitaraman, M. Potsdam, B. Jayaraman, A. Datta, A. Wissink, D. Mavriplis, H. Saberi, Rotor loads prediction using Helios: A multi-solver framework for rotorcraft CFD/CSD analysis, AIAA Paper 2011-1123 (Jan. 2011).

${ }^{7}$ M. J. Smith, J. W. Lim, B. G. van der Wall, J. D. Baeder, R. T. Biedron, D. D. Boyd Jr., B. Jayaraman, S. N. Jung, B.-Y. Min, The HART II International Workshop: An assessment of the state of the art in CFD/CSD prediction, CEAS Aeronautical Journal 4 (2013) 345-372.

${ }^{8}$ R. Celi, Recent applications of design optimization to rotorcraft - a survey, Journal of Aircraft 36 (1999) 176-189.

${ }^{9}$ J. L. Walsh, K. C. Young, F. J. Tarzanin, J. E. Hirsh, D. K. Young, Optimization issues with complex rotorcraft comprehensive analysis, AIAA Paper 1998-4889 (Sept. 1998).

${ }^{10}$ E. J. Nielsen, B. Diskin, Discrete adjoint-based design for unsteady turbulent flows on dynamic overset unstructured grids, AIAA Journal 51 (6) (2013) 1355-1373.

${ }^{11}$ A. Mishra, K. Mani, D. Mavriplis, J. Sitaraman, Time dependent adjoint-based optimization for coupled fluid-structure problems, Journal of Computational Physics 292 (2015) 253271.

${ }^{12}$ R. T. Biedron, J.-R. Carlson, J. M. Derlaga, P. A. Gnoffo, D. P. Hammond, W. T. Jones, B. Kleb, E. M. Lee-Rausch, E. J. Nielsen, M. A. Park, C. L. Rumsey, J. L. Thomas, W. A. Wood, FUN3D Manual: 13.2, NASA/TM-2017-219661, NASA Langley Research Center, Hampton, VA (2017).

${ }^{13}$ J. C. Newman III, W. K. Anderson, D. L. Whitfield, Multidisciplinary sensitivity derivatives using complex variables, Computational Fluid Dynamics Laboratory, NSF Engineering Research Center for Computational Field Simulation, MSSUCOE-ERC-98-08, 1998 (July 1998).

${ }^{14}$ L. Wang, B. Diskin, R. T. Biedron, E. J. Nielsen, O. A. Bauchau, Sensitivity analysis of multidisciplinary rotorcraft simulations, AIAA Paper 2017-1670 (Jan. 2017). 
${ }^{15}$ L. Wang, B. Diskin, R. T. Biedron, E. J. Nielsen, O. A. Bauchau, High-fidelity multidisciplinary sensitivity analysis and design optimization for rotorcraft applications, AIAA Journal, Submitted (Sept. 2017).

${ }^{16}$ V. Sonneville, A. Cardona, O. Brüls, Geometrically exact beam finite element formulated on the special Euclidean group SE(3), Computer Methods in Applied Mechanics and Engineering 268 (2014) 451-474.

${ }^{17}$ V. Sonneville, O. Brüls, A formulation on the special Euclidean group for dynamic analysis of multibody systems, Journal of Computational and Nonlinear Dynamics 9 (4) (2014) 041002.

${ }^{18}$ V. Sonneville, O. Brüls, O. Bauchau, Interpolation schemes for geometrically exact beams: A motion approach, International Journal of Numerical Methods in Engineering. To appear.

${ }^{19}$ E. J. Nielsen, B. Diskin, High-performance aerodynamic computations for aerospace applications, Parallel Computing 64 (2017) 20-32.

${ }^{20}$ V. Vatsa, M. H. Carpenter, D. Lockard, Re-evaluation of an optimized second order backward difference (BDF2OPT) scheme for unsteady flow applications, AIAA Paper 20100122 (Jan. 2010).

${ }^{21}$ R. Noack, DiRTlib: A library to add an overset capability to your flow solver, AIAA Paper 2005-5116 (June 2005).

${ }^{22}$ R. Noack, D. Bogar, R. Kunz, P. Carrica, SUGGAR++: An improved general overset grid assembly capability, AIAA Paper 2009-3992 (June 2009).

${ }^{23}$ W. K. Anderson, J. C. Newmann, D. L. Whitfield, E. J. Nielsen, Sensitivity analysis for Navier-Stokes equations on unstructured meshes using complex variables, AIAA Journal 39 (1) (2001) 56-63.

${ }^{24}$ J. R. R. A. Martins, I. M. Kroo, J. J. Alonso, An automated method for sensitivity analysis using complex variables, AIAA Paper 2000-0689 (Jan. 2000).

${ }^{25}$ J. N. Abras, C. E. Lynch, M. J. Smith, Computational Fluid Dynamics-Computational Structural Dynamics rotor coupling using an unstructured Reynolds-averaged NavierStokes methodology, Journal of The American Helicopter Society 57 (012001) (2012) 1-14.

${ }^{26}$ E. J. Nielsen, W. K. Anderson, Recent improvements in aerodynamic design optimization on unstructured meshes, AIAA Journal 40 (6) (2002) 1155-1163.

${ }^{27}$ P. E. Gill, W. Murray, M. A. Saunders, SNOPT: An SQP algorithm for large-scale constrained optimization, SIAM Journal on Optimization 12 (4) (2002) 979-1006.

${ }^{28}$ P. E. Gill, W. Murray, M. A. Saunders, SNOPT: An SQP algorithm for large-scale constrained optimization, SIAM Review 47 (1) (2005) 99-131.
${ }^{29}$ A. A. Kumar, S. R. Viswamurthy, R. Ganguli, Correlation of helicopter rotor aeroelastic response with HART II wind tunnel test, Aircraft Engineering and Aerospace Technology 82 (4) (2010) 237-248.

${ }^{30} \mathrm{~J}$. W. Lim, An assessment of rotor dynamics correlation for descending flight using CSD/CFD coupled analysis, 64th Annual Forum, American Helicopter Society, Montreal, Canada (April-May 2008).

${ }^{31}$ J. A. Samareh, Novel multidisciplinary shape parameterization approach, Journal of Aircraft 38 (6) (2001) 1015-1024. 